\title{
Severe left flank pain
}

\author{
Haneen Mallah MD, John Makram MD, Thanita Thongton MD
}

\section{CASE}

A 43-year-old woman who was an active smoker with a history of pulmonary embolism in 2015 (attributed to smoking and oral contraceptives managed with 6 months of oral anticoagulation) was transferred from an outside facility for evaluation after she presented with severe left flank pain described as a constant, crushing pain that started acutely three days prior to admission. Computed tomography (CT) with angiography of abdomen/pelvis showed a partially thrombosed splenic artery aneurysm $(12 * 12 \mathrm{~mm})$ and a superior medial splenic infarct less than $50 \%$ of splenic volume (Figures 1 and 2). Hypercoagulability workup, including rheumatoid factor, C3 and C4, ANA, dsDNA, scleroderma antibodies, C-ANCA, P-ANCA, anti-cardiolipin antibody, factor $\checkmark$ Leiden, and prothrombin mutation, was negative. Her transthoracic echo was also unremarkable.

\section{Discussion}

Splenic artery aneurysm is 4 times more common in women than in men with a proposed incidence of
$0.8 \%$ based on angiographic studies. The least frequent complication of splenic aneurysm is splenic infarction. Since the spleen is supplied by both the splenic artery and the short gastric arteries, it has collateral circulation when an artery is occluded, resulting in an incomplete infarct in the majority of cases. In a retrospective review of 32 cases of splenic infarcts at a single institution over a 10-year period, the most common cause was a cardioembolic event (63\%). Other causes included valvular heart disease without atrial fibrillation, systemic lupus erythematosus with antiphospholipid syndrome, and infective endocarditis. The underlying cause was known in two-thirds of the patients at the time splenic infarction occurred; in the others, it was diagnosed following splenic infarction. ${ }^{1}$ In a retrospective review of 59 cases of splenic infarction, the most common causes were hematologic and prothrombotic disorders, which accounted for 59 and 29 percent of cases, respectively. ${ }^{2}$ The most common presenting findings include pain, fever, nausea or vomiting, and splenomegaly. . $^{3,4}$

Abdominal pain can be accompanied by shivering, pleuritic chest pain, and left shoulder pain (Kehr's sign

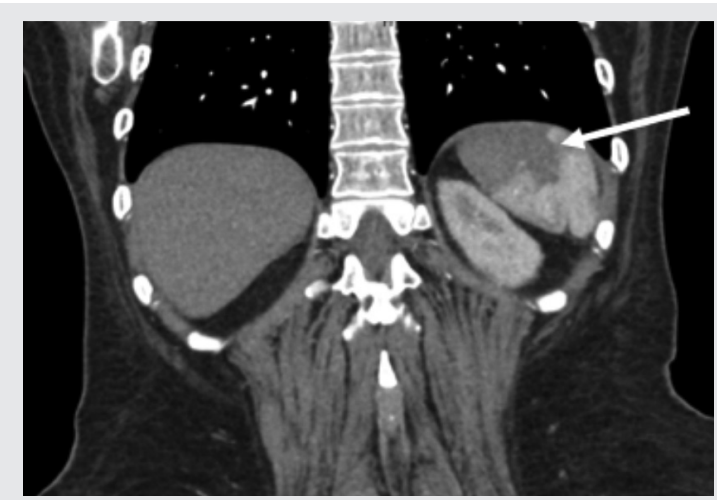

Figures 1 and 2. A wedge-shaped hypodensity seen within the spleen that extends superiorly. The white arrow points to the infarct.

Corresponding author: Haneen Mallah

Contact Information: Haneen.mallah@ttuhsc.edu

DOI: $10.12746 /$ swrccc.v8i34.687 
of referred pain). Computed tomography with contrast is the best method for the diagnosis of splenic infarct. It is also more useful than other diagnostic methods for the identification of other pathologies. Magnetic resonance imaging with intravenously injected gadolinium contrast medium is another option.5,6 The possibility of splenic infarct should be considered in patients at risk and with non-specific left upper quadrant pain. Management of splenic infarction depends upon the underlying cause; appropriate analgesics are usually required for pain control in the majority of cases. Anticoagulation is generally appropriate if the underlying cause is a hypercoagulable state, such as antiphospholipid syndrome, heparin-induced thrombocytopenia, a cardioembolic source, or an inherited thrombophilia. If an underlying cause cannot be identified (i.e., truly cryptogenic infarction), anticoagulation is still likely a prudent option.

Keywords: LUQ pain, splenic infarct, hypercoagulable state, splenic artery aneurysm

Article citation: Mallah $\mathrm{H}$, Makram J, Thongton T. Severe left flank pain. The Southwest Respiratory and Critical Care Chronicles 2020;8(34):77-78

From: Department of Internal Medicine, Texas Tech University Health Sciences Center, Lubbock, Texas

Submitted: $2 / 24 / 2020$

Accepted: $3 / 27 / 2020$

Reviewer: Kenneth Nugent MD

Conflicts of interest: none

This work is licensed under a Creative Commons

Attribution-ShareAlike 4.0 International License.

\section{REFERENCES}

1. Schattner A, Adi M, Kitroser E, et al. Acute splenic infarction at an academic general hospital over 10 years: presentation, etiology, and outcome. Medicine (Baltimore); 2015;94(36): e1363. doi: 10.1097/MD.0000000000001363.

2. Nores M, Phillips EH, Morgenstern L, et al. The clinical spectrum of splenic infarction. Am Surg 1998;64:182-88.

3. Lawrence YR, Pokroy R, Berlowitz D, et al. Splenic infarction: an update on William Osler's observations. Isr Med Assoc J 2010;12:362-65.

4. Sridhar S, Lau SK, Woo PC. A diagnostic pitfall: salmonella splenic infarction in hereditary spherocytosis. Am J Med 2016; 129:42-43.

5. Kamaya A, Weinstein S, Desser TS. Multiple lesions of the spleen: differential diagnosis of cystic and solid lesions. Semin Ultrasound CT MR 2006;27:389-403.

6. Thipphavong S, Duigenan S, Schindera ST, et al. Nonneoplastic, benign, and malignant splenic diseases: cross-sectional imaging findings and rare disease entities. AJR Am J Roentgenol 2014;203:315-22. 